the book. Let me pick out a few about which I have some independent knowlledge. The long section on quantum measurement theory emphasizes the many dilemmas and queries that one encounters if one assumes, with Bohr, that there is a genuine dichotomy between the microscopic world in which quantum theory applies and the macroscopic world of measurement apparatus. These mindbending difficulties ('EPR', 'entanglement', Bell's theorem and so on) are the stuff of rather boring philosophical discussions; it is hard to see how they could make consciousness easier to understand. But one seems unable to find any natural scale for this dichotomy, among other things; and many, if not most, thinking quantum physicists reject the idea that there is any dichotomy, and assume that quantum laws hold all the way up and down. This possibility is dismissed by Penrose in two brief pages (pp. 310-312).

Penrose's primary objection to this point of view is that it is "unsatisfactory" in that it involves continual splitting of the wave function of the Universe into fragments, only one of which an observer can perceive. (This splitting is the 'manyworlds' viewpoint, although there are other ways to interpret the same mathematics, among them that of M. Gell-Mann and J. Hartle.) We cannot decide for nature which of her ways are 'satisfactory' or 'unsatisfactory'; that is nature's call.

More seriously, Penrose makes the claim that there is no quantitative justification for the all-quantum viewpoint. In a popular book, The Quark and the Jaguar, published earlier this year, as well as in several articles, Gell-Mann discusses at length the rapid and complete 'decoherence' between alternatives, which prevents the observation of coexistence within, for a typical case, $10^{-21}$ seconds, by actual and precise calculation. That this is a consistent and logically satisfactory possibility has been obvious for many years, since. Fritz London first proposed it in 1938 It has now been formalized. Penrose should have been aware of this.

With regard to superconductivity, Penrose has, I think, got the implications of macroscopic quantum coherence backwards. In a superconductor, the quantum field itself becomes a macroscopic object, a perfectly measurable, rigid, thermodynamic parameter of the body on the same footing as strain, torque, entropy or magnetization, and obeying the same general laws (which derive from the general phenomenon of broken symmetry). Coherence is maintained not by an energy gap as Penrose suggests, or by some mysterious persistence of a quantum superposition, but by mundane thermal equilibrium. It has always seemed to me that for anyone in possession of the facts about superfluidity and superconductivity, it would be hard to doubt that classical behaviour is simply large-scale quantum behaviour - that is, an emergent property of large quantum systems. But habits of thought die hard.

Microtubules are for Penrose the likely seat of the mysterious quantum gravitational effect that makes the mind possible. Biophysicists who specialize in their study would agree that the behaviour of microtubules is indeed interesting and complex, but would see no need (nor in fact any room) for anything but the characteristic chemical control mechanisms with which we are familiar.

Penrose has written a complex, erudite and fascinating book, and my complaints about it do not mean that I did not enjoy and learn a great deal from reading it. But one should keep in mind that Penrose is a mathematician with little experience of the messy, frustrating but ultimately deeply satisfying process of checking his ideas against the experimental facts about nature. Mathematicians are used to gameplaying according to a set of rules they lay down in advance, despite the fact that nature always writes her own. One acquires a great deal of humility by experiencing the real wiliness of nature.

P. W. Anderson is in Department of Physics, Jadwin Hall, Princeton University, Princeton, New Jersey 08544, USA.

\section{Mental pictures on the brain}

\section{Zenon Pylyshyn}

Image and Brain: The Resolution of the Imagery Debate. By Stephen M. Kosslyn. MIT Press: 1994. Pp. 516. \$45, £40.50.

THIs book is intended first and foremost as the final solution to the so-called 'imagery debate'. Its focus on this polemical task, however, seriously detracts from its potential usefulness as a study of the relation between visualization and vision, particularly from the perspective of clinical neurology.

Many of us believed that the debate, at least in the form revived in this book, had quietly disappeared as it became clear that there were serious problems with notions such as that mental images 'depict' or 'resemble' something or 'have spatial properties'. But Stephen Kosslyn now feels that these notions can be rehabilitated because "by turning to the brain, this debate can be resolved to the satisfaction of most people". But the basic problem still stands: as long as the research questions continue to be ill-posed, the problem about mental images will remain unsolved, regardless of how much brain (or other) data is collected.
Discussions of the nature of mental imagery have invariably equivocated between two very different views of what an image is. The literal option is that an image is some sort of mapping (usually viewed as a quasi-photographic projection) of the imagined scene onto some real (presumably neural) surface, possessing such physical and/or geometrical properties as shape, length, area and size. I do not know anyone who explicitly endorses this literal 'picture' option. In a way this is too bad because it is the only option that actually addresses much of Kosslyn's data (such as the increased time it takes to scan greater imagined distances or to examine smaller images). It is also the only option that clearly connects with most of the neurological findings discussed in the book. (Imagining something large, for example, results in brain activity over a larger area of cortex than imagining something small.)

The second option is that we have some 'functional equivalent' of pictures in our brain. Kosslyn talks about a "functional space" where images do not actually 'have' properties such as size or orientation but merely 'specify' them. But this option has no explanatory power because it fails to constrain the nonliteral 'image' to have any particular intrinsic properties - gone are depiction and resemblance, as are any constraints on how geometrical properties are represented. To account for empirical data one must of course reintroduce whatever additional constraints one needs, but these are no longer intrinsic properties of the image. As Kosslyn remarks, the critical properties are not inherent in the image but in how it is 'read'. Moreover, since such a functional image contains "previously digested information", there is no reason why 'reading' it should involve the visual system.

A good example of this sort of extrinsic stipulation of constraints is Kosslyn's use of a matrix as a functional image in his computer model. Notice that a matrix, by virtue of being a data structure, does not require scanning to proceed through adjacent cells, nor does it inherently preserve geometrical properties over transformations such as translation and rotation. Such constraints must be additionally stipulated. Consequently, appealing to the matrix itself does not explain predictions derived from such stipulated constraints, as it would if we had taken the literal option and assumed a surface constrained by the laws of physics.

The basic problem is that any theory of mental imagery has two fundamental degrees of freedom between which it can trade off in addressing the data, since the theory specifies both the nature of the image and the nature of the process that examines it. If we assume a literal view of the image, the physical geometry of the display allows us to make sense of some 
behavioural and brain data, but only if we also assume a literal 'mind's eye' that is very much like a real eye, complete with a pattern of spatial resolution, a visual angle and a way of scanning with mental 'eyemovements'. On the other hand, if we take the nonliteral option, then empirical results otherwise attributed to the geometry of the image and the 'mind's eye' must now be built into the interaction between the image and the access process. Because there are no independent constraints on such a process, we are free to tailor it to fit the data. But we do pay a price because now there is nothing left of the idea of a depictive, geometry-preserving pictorial entity, and so there is no need to involve the visual system.

Kosslyn's book is at its best when it skirts the question of the nature of images and focuses instead on the commonality of vision and visual imagery. Both behavioural and neural evidence is presented that suggests that vision and imagery may use common brain mechanisms. Although the evidence is far from unambiguous (especially evidence from the superposition of images and percepts, for which there is a simple attention-based 'indexing' explanation) it is nonetheless intriguing and worth examining in detail. On the other hand, this approach belies the goal of the book: it is irrelevant to the debate about the nature of images.

Nobody doubts that some brain mechanisms are involved in both vision and imaging. The interesting question is which mechanisms they share, because the answer might illuminate what, if anything, is special about visualizing as opposed to general reasoning. If it could be shown that imagery uses mechanisms that are specific to vision, then we would have discovered something of considerable interest - even though it would still not address the traditional 'imagery debate'. Mechanisms specific to vision might, for example, include those responsible for the segregation of figure from ground, spontaneous construction of three-dimensional percepts from two-dimensional contours or motion cues, or any of the many distinctly visual phenomena described in textbooks on visual perception. Kosslyn's book provides no unproblematic evidence implicating this kind of mechanism. What it provides instead is some speculation about why positing internal pictures helps to explain perceptual invariances and the integration of information from glances - speculation that helps only to perpetuate the misleading subjective experience we have that when we see or imagine something we are creating a replica of it in our heads, an idea that has surely run its course over the past two millennia.

Zenon Pylyshyn is in the Center for Cognitive Science, Rutgers University, New Brunswick, New Jersey 08903, USA.
Minds possessed

\section{Susan Blackmore}

Dark White: Aliens, Abductions and the UFO Obsessions. By Jim Schnabel. Hamish Hamilton: 1994. Pp. 304. £16.99.

How can apparently sane, intelligent and likeable people believe that four-foot-high aliens are visiting our planet and abducting people? If you are perplexed by this question, Jim Schnabel's latest book Dark White (Grey - geddit?) will give you some answers.

A recent Roper opinion poll claimed that nearly four million Americans have been abducted. The stories are remarkably consistent as well as outrageous. People are woken in the dead of night or, less commonly, taken from their car or workplace, and confronted by large-headed, small-bodied, huge-eyed grey aliens who transport them magically into a spacecraft. Here they are taken down curved corridors, laid on a flat table and subjected to humiliating or terrifying mental, medical and gynaecological procedures. Eventually they find themselves back in bed but with two or three hours 'missing'.

The stories cry out for comparison with fairy abductions, incubi and succubi and myths such as the Old Hag of Newfoundland, who visits victims at night and tries to suffocate them. Schnabel deals well with all of these but his greatest strength lies in the way he portrays the main characters involved.

Take Budd Hopkins, a New York artist who first saw a UFO in 1964 . He began to investigate experiences of 'missing time' and found himself overwhelmed by people needing help. Perhaps it was because he was already well known as an artist that people took him seriously. He learned how to hypnotize them and soon they were 'remembering' the abductions that took place in the missing time intervals.

Schnabel portrays Hopkins as a kindly and sincere man who really wanted to understand what was going on. The vividness and consistency of the stories persuaded him of the nuts-and-bolts reality of the aliens and their UFOs. It was also Hopkins who first came across stories of the alien hybridization programme. As Schnabel points out, as soon as Budd recognized it, women began turning up with strange scars and tales of disappearing pregnancies, and men told of having sperm removed by beautiful female aliens.

Contrasting with the sincerity of a possibly naive artist is the craziness of Whitley Strieber. The way Schnabel describes him you would not trust his opinion of what he had for breakfast, let alone the reality of his aliens. His best-selling book Communion followed several horror nov- els that, according to Schnabel, mix fiction and biography with alarming ease - and apparently it was going to be called Body Terror until Strieber decided that it must not frighten people.

This tension between terror and enlightenment runs through the whole story. Whereas Hopkins' abductees seemed only to experience pain and fear, those studied by Leo Sprinkle, a psychologist from Wyoming, more often reported spiritual experiences and "environmental and spiritual consciousness-raising". Joining this clan was soon to be John Mack, well known as a Pulitzer prizewinning author and professor of psychiatry at Harvard University. With such qualifications he had an authority perhaps greater than any of the others involved. He soon collected a large following of abductees convinced that the aliens had peaceful intent and wanted to warn us of impending environmental disaster.

Like Mack, Ken Ring, a psychologist, sees the positive side. He had studied near-death experiences and noted the similarities between these and abductions. Both pointed to the progress of human consciousness towards unity and harmony.

A problem for anyone who simply wants to know whether or not the aliens exist is that these academics, unlike the more down-to-earth Hopkins, can evade such a crude question: after all, it depends on what one means by reality. But what of the science?

There is certainly a scientific story to be told. Yet, with no index or proper references, this book fails to tell it well enough. This is a shame because the groundwork is all there. Schnabel tackles sleep paralysis, in which the muscle paralysis of dreaming sleep can carry over into waking, and compares it with abduction myths. He considers childhood trauma, the problems of hypnosis and the arguments over false memory.

Best of all he clearly explains the most tricky - and interesting - of the theories. Michael Persinger, a Canadian neuroscientist, argues that the experiences are caused by firing in the temporal lobes of the brain and can be set off by changes in magnetic fields. Schnabel marshals the evidence for this theory and concludes that abduction accounts may all be similar not because the aliens are all similar but because our brains are. Stimulation of the relevant areas, combined with cultural and personal material, may account for it all.

The book convinced me that abduction accounts are well worth serious research. It is not a question of whether or not the aliens exist but of what the experiences have to tell us about our minds and brains.

Susan Blackmore is in the Department of Psychology, University of the West of England, Bristol BS16 2JP, UK. 